\title{
Distributed Joint Source-Channel Coding for Correlated Sources Using Non-systematic Repeat-Accumulate Based Codes
}

\author{
Valtteri Tervo • Tad Matsumoto • Pen-Shun Lu
}

Published online: 27 March 2012

(C) The Author(s) 2012. This article is published with open access at Springerlink.com

\begin{abstract}
In this paper, we propose a technique for coding the data from multiple correlated binary sources, with the aim of providing an alternative solution to the correlated source compression problem. Using non-systematic repeat-accumulate based codes, it is possible to achieve compression which is close to the Slepian-Wolf bound without relying on massive puncturing. With the technique proposed in this paper, instead of puncturing, compression is achieved by increasing check node degrees. Hence, the code rate can be more flexibly adjusted with the proposed technique in comparison with the puncturing-based schemes. Furthermore, the technique is applied to distributed joint source-channel coding (DJSCC). It is shown that in many cases tested, the proposed scheme can achieve mutual information very close to one with the lower signal-to-noise power ratio than turbo and low density generator matrix based DJSCC in additive white Gaussian noise channel. The convergence property of the system is also evaluated via the extrinsic information transfer analysis.
\end{abstract}

Keywords Concatenated codes - Cooperative coding - Iterative decoding - EXIT chart

\section{Introduction}

Distributed source coding (DSC) and joint source-channel coding (JSCC) have been very active research areas in recent years. Lossy and lossless source coding for correlated sources is one of the hottest research topics nowadays. The Slepian-Wolf (SW) theorem [1] states that the joint entropy of the sources is achievable as long as decoding is performed jointly, while encoding can be performed independently. Conventional source coding under a distortion constraint and channel coding under the resource constraints of the channels, such as power and/or spectrum availability, have long been considered as the information-theoretic duals

\footnotetext{
V. Tervo $(\varangle) \cdot$ T. Matsumoto $\cdot$ P.-S. Lu

Centre for Wireless Communications, University of Oulu, P.O. Box 4500, 90014 Oulu, Finland

e-mail:wade@ee.oulu.fi

V. Tervo $\cdot$ T. Matsumoto $\cdot$ P.-S. Lu

Japan Advanced Institute of Science and Technology, 1-1 Asahi-Dai, Nomi, Ishikawa 923-1292, Japan
} 
of each other, starting from Shannon's 1959 paper [2]. In [3], an exact characterization of the Shannon duality between data transmission and compression through Lagrange duality in convex optimization is provided. This suggests that excellent channel codes may also be excellent for the source compression.

In [4], A. Wyner realized the close connection of DSC to channel coding, and suggested the use of linear channel codes as a constructive approach to SW coding (SWC). Garcia-Frias et al. propose the use of turbo codes [5] for the compression of correlated binary sources in [6]. They applied the same structure for noisy channels in [7]. The use of linear codes with low density generator matrix (LDGM) for channel coding, compression, and JSCC of correlated sources was proposed in [8]. A more advanced low complexity cooperative source-channel coding scheme based on the proper use of LDGM code was proposed in [9]. In [10] a constructive approach for DSC of correlated binary sources using linear channel codes that can achieve any point of the SW region was proposed. A good tutorial for DSC for sensor networks is given in [11]. The applicability of DSC in video coding is investigated in $[12,13]$. A universal approach to source controlled channel decoding was proposed in [14].

Most of the current practical SW codes, which are based on the use of channel codes, utilize puncturing for the rate adjustment. In this paper, we present an alternative technique to the puncturing based turbo compression. An advantageous point of the proposed technique over the conventional puncturing-based compression is that no information bits are lost, while they may be lost with the puncturing-based compression, of which category the conventional techniques belong to. Furthermore, we use our proposed technique to distributed JSCC (DJSCC) of correlated sources. We focus only on two-source symmetric scenarios, i.e., channels' statistical properties are identical for the two sources.

Repeat-accumulate (RA) [15] codes can be thought as a special case of low-density parity-check (LDPC) codes [16]. It has been proven in [17] that, in theory, RA codes can achieve the Shannon capacity in point-to-point additive white Gaussian noise (AWGN) channels. We utilize such powerful and flexible properties of RA codes for the compression of correlated sources and further apply the structure to DJSCC. The use of non-systematic code is beneficial because it can achieve arbitrary rate pairs of the signal to be transmitted, which is not always possible with systematic code. As described in [7], with turbo DJSCC, a half of the systematic bits are transmitted from the first encoder, and the other half from the second one. This explicitly indicates that one has to be very careful when determining the right puncturing pattern. Unlike the conventional approach, puncturing is not necessary in our proposed system. We set the average check node degree in RA code to be larger than the average variable node degree. In that way, we get the channel code rate larger than one and, hence, compression is achieved.

In order to understand the convergence properties of the system, we perform the extrinsic information transfer (EXIT) analysis [18] for our proposed system. This is motivated by the area property, which states that the area of the tunnel between the EXIT curves of the constituent decoders of a concatenated system is proportional to the gap between the source coding rate and the entropy.

The main contributions of this paper are summarized as follows. We propose a symmetric technique for coding the data from two correlated binary sources by using non-systematic RA based codes. The rate allocation can be performed via the node degree allocation and, hence, no puncturing is needed. Furthermore, we propose a doped accumulator (ACC) to shape the EXIT curves for better matching between them. Finally, we provide mathematical derivation for the relationship between the EXIT curves of the component decoders in the case that the code rate is smaller than or equal to 1 . 
The rest of this paper is organized as follows. Sections 2.1 and 2.2 describe the proposed system, with an emphasis on the structures of the encoder and decoder, respectively. Section 2.3 characterizes the doping technique used in [19]. In Sect. 3, the extrinsic information exchange property is analyzed theoretically. In Sect. 4, we demonstrate the performance of the system through EXIT chart analysis. Furthermore, we compare the performance of our proposed technique with counterpart techniques based on symmetric turbo code presented in $[6,7]$ and LDGM code presented in [8]. Finally, Sect. 5 concludes this paper with some remarks.

\section{Proposed System}

In this section, the structures of the proposed encoder and decoder are described.

\subsection{Encoder}

The encoder structure is shown in Fig. 1 . The encoder chain VNE- $\Pi_{s}-\mathrm{CNE}$ with $s=1,2$, where VNE stands for variable node encoder [20] and CNE stands for check node encoder [20], can be viewed as a special case of LDPC code. Since the standard LDPC code cannot achieve desirable performance in terms of compression when the correlation between the two sources is low, we attach ACC before the LDPC encoder. Furthermore, when the signal to noise power ratio (SNR) is low, we need to attach another ACC after the encoder to achieve better performance in terms of bit error ratio (BER). When the both ACCs are attached, the code is equivalent to accumulate-repeat-accumulate (ARA) code [21].

The information sequence $\mathbf{U}^{\mathrm{s}}=u_{1}^{s}, u_{2}^{s}, \ldots, u_{L}^{s}$ is optionally accumulated and outer perfect doping [19] is used for the binary sequence output of the source. A pseudorandom doping sequence $\mathbf{D}=d_{1}, d_{2}, \ldots, d_{L\left(1-R_{D}\right) / R_{D}}$, where $R_{D}\left(R_{D} \leq 1.0\right)$ is the code rate for doping and $d_{k} \in\{0,1\}$, is placed pseudorandomly into the accumulated sequence resulting
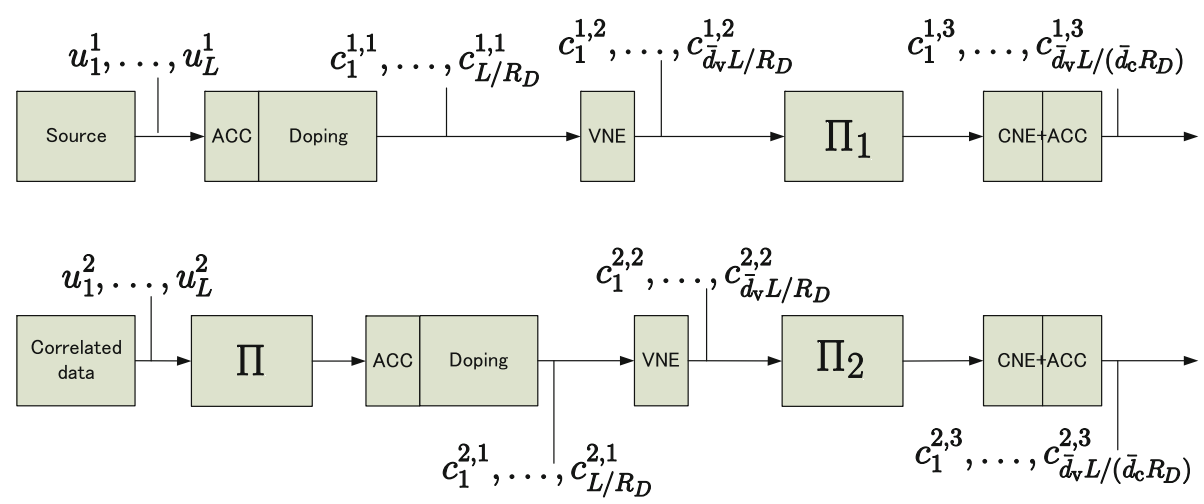

Fig. 1 Transmitter side of the system model. Each source is encoded by an RA based encoder. The first ACC is used only when correlation is low and the second ACC is used when SNR is low. Encoders are enumerated as follows: $\left\{c_{k}^{s, 1}\right\}$ represents the coded bits for source $s$ after the first ACC and doping. $\left\{c_{k}^{s, 2}\right\}$ are the coded bits for the source $s$ associated to VNE. $\left\{c_{k}^{s, 3}\right\}$ represents the coded bits for source $s$ associated to encoder $\mathrm{CNE}+\mathrm{ACC}$ which consist of a check node encoder and an accumulator. $\Pi$ is a random interleaver of length $L / R_{D}$ and $\Pi_{1}$ and $\Pi_{2}$ are random interleavers of length $\bar{d}_{\mathrm{V}} L / R_{D}$ 
in a sequence $\mathbf{C}^{s, 1}=c_{1}^{s, 1}, c_{2}^{s, 1}, \ldots, c_{L / R_{D}}^{s, 1}$. D and its position indices in the vector $\mathbf{C}^{s, 1}$ is assumed to be known to the decoder. The doping sequence $\mathbf{D}$ is used to improve the convergence properties of the system.

The correlated data is modeled according to the bit flipping model, i.e., $u_{k}^{2,1}=u_{k}^{1,1} \oplus e_{k}$ $k=1,2, \ldots, L$, where $\oplus$ indicates the modulus 2 addition and $e_{k}$ is a random variable, which takes value 1 with probability $p$ and 0 with probability $1-p$. Both sequences $\mathbf{U}^{s}$, $s=1,2$, are encoded independently each other by using non-systematic RA or ARA code with doping. The RA code consists of VNE, CNE and ACC. After the coding, binary phase shift keying (BPSK)-modulated symbols are sent trough the AWGN channel with the noise variance $\sigma_{n}^{2}$.

A factor graph of ARA encoder with doping is depicted in Fig. 2. First of all, the source sequence $\mathbf{U}^{S}$ is passed trough the ACC which consists of $L$ check nodes. Note that all the output bits depends on all the bits preceding it. After the first ACC, the doping sequence $\mathbf{D}$ is included and the resulting sequence is passed through $L / R_{D}$ variable nodes. The resulting sequence is interleaved and provided to CNE which consists of check nodes. Finally, the sequence is accumulated again and passed forward to the BPSK modulator.

Let $D_{\mathrm{v}}$ be the number of different variable node degrees, and denote their degrees by $\tilde{d}_{\mathrm{v}, i}$, $i=1, \ldots, D_{\mathrm{v}}$. The average variable node degree is calculated as [20]

$$
\bar{d}_{\mathrm{v}}=\sum_{i=1}^{D_{\mathrm{v}}} a_{\mathrm{v}, i} \tilde{d}_{\mathrm{v}, i},
$$

where $a_{\mathrm{v}, i}$ is the fraction of nodes having degree $\tilde{d}_{\mathrm{v}, i}$. In the same way, average check node degree is calculated as [20]

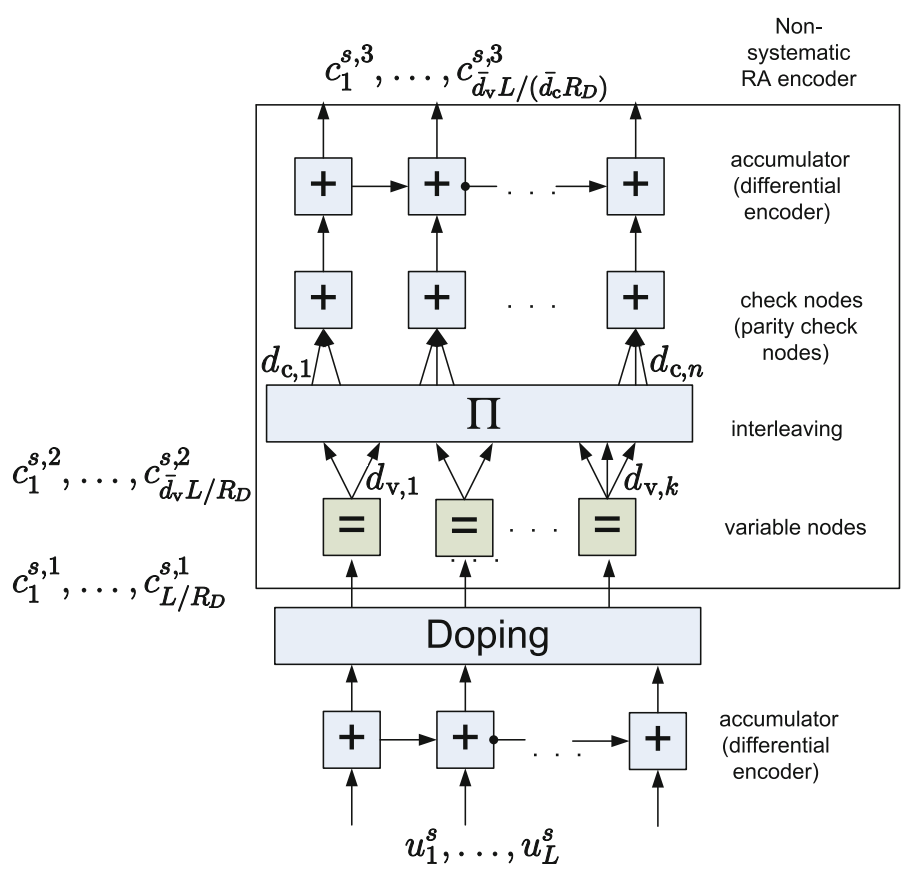

Fig. 2 Factor graph of non-systematic ARA encoder with doping 


$$
\bar{d}_{\mathrm{c}}=\sum_{i=1}^{D_{\mathrm{c}}} a_{\mathrm{c}, i} \tilde{d}_{\mathrm{c}, i} .
$$

The overall code rate of the ARA code is

$$
R_{\mathrm{c}}^{\mathrm{ARA}}=\frac{\bar{d}_{\mathrm{c}}}{\bar{d}_{\mathrm{v}}} .
$$

If $\bar{d}_{\mathrm{c}}>\bar{d}_{\mathrm{v}}$, then $R_{\mathrm{c}}^{\mathrm{ARA}}>1$, and, hence, it achieves compression. The data can be reconstructed in the receiver with the help of a priori information provided by the other decoder.

When we use doping, the overall code rate becomes

$$
R_{\mathrm{c}}=R_{D} \frac{\bar{d}_{\mathrm{c}}}{\bar{d}_{\mathrm{v}}},
$$

and the compression is achieved when $\bar{d}_{\mathrm{c}}>R_{D} \bar{d}_{\mathrm{v}}$.

\subsection{Decoder}

The extrinsic information exchange takes place in the decoder as depicted in Fig. 3. $f_{\mathrm{c}}$ denotes the extrinsic information update function due to the correlation, which is given by

$$
\begin{aligned}
\lambda_{A}^{q, j} & =f_{\mathrm{c}}\left(\lambda_{E}^{s, j}\right) \\
& =\ln \frac{(1-p) e^{\lambda_{E}^{s, j}}+p}{(1-p)+p e^{\lambda_{E}^{s, j}},}
\end{aligned}
$$

where $\lambda_{E}^{s, j}$ is the extrinsic log-likelihood ratio (LLR) of the $j$ th bit provided by the decoder

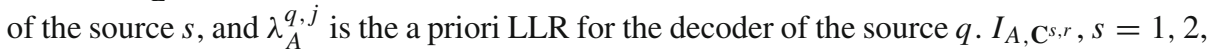
$r=1,2,3$, denotes the a priori information for the decoder $r$ of the source $s . I_{E, \mathbf{C}^{s, r} \text { denotes }}$ the extrinsic information provided by the decoder $r$ of the source $s . I_{A^{\prime}, \mathbf{C}^{s, r}}$ and $I_{E^{\prime}, \mathbf{C}^{s, r}}$ correspond to $I_{A}, \mathbf{C}^{s, r}$ and $I_{E, \mathbf{C}^{s, r}}$, respectively, however, it should be noticed that the information is forwarded towards the opposite direction. $I_{A_{\mathbf{D}}^{\prime}}$ denotes the mutual information (MI) provided

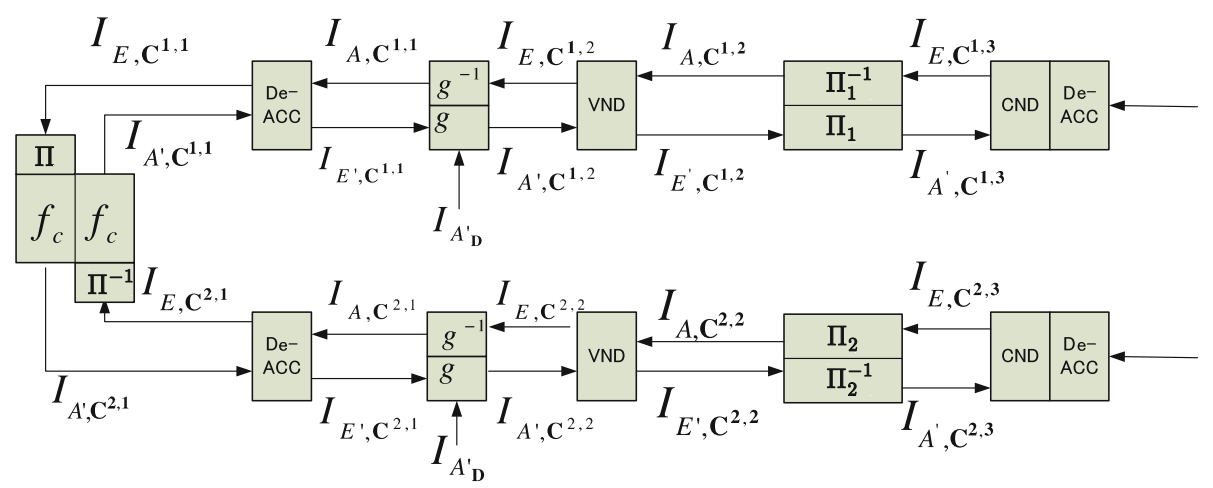

Fig. 3 Decoder structure showing the exchange of extrinsic information for the proposed system. $g$ denotes the EXIT function corresponding to the LLR addition $\lambda_{E^{\prime}, \mathbf{C}^{\mathbf{1}, \mathbf{2}}}+\lambda_{A_{\mathbf{D}}^{\prime}}$ or $\lambda_{E^{\prime}, \mathbf{C}^{\mathbf{2}, \mathbf{1}}}+\lambda_{A_{\mathbf{D}}^{\prime}}$. VND and CND denote variable node decoder and check node decoder, respectively. DeACC denotes de-accumulator that decodes the ACC coded data 
as a result of outer perfect doping and $g$ denotes the EXIT function corresponding to the LLR addition $\lambda_{E^{\prime}, \mathbf{C}^{\mathbf{1}, 2}}+\lambda_{A_{\mathbf{D}}^{\prime}}$ or $\lambda_{E^{\prime}, \mathbf{C}^{\mathbf{2}, 1}}+\lambda_{A_{\mathbf{D}}^{\prime}}$, where $\lambda_{A_{\mathbf{D}}^{\prime}}=\lambda_{A_{d_{1}}^{\prime}}, \lambda_{A_{d_{2}}^{\prime}}, \ldots, \lambda_{A_{d_{L / R}}^{\prime} \mathbf{D}}$ denotes the LLR sequence including the doped bits defined as

$$
\lambda_{A_{d_{k}}^{\prime}}= \begin{cases}\operatorname{sign}\left(c_{k}^{1,1}\right) \cdot m & \text { if } c_{k}^{1,1} \text { is doped bit } \\ 0 & \text { otherwise, }\end{cases}
$$

where $m$ is a pre-set large value. All the numerical calculations are performed in the LLR domain to avoid the numerical instability and to increase the range of the likelihood values.

Note that there are two inputs into the decoder chain. To identify that information can be exchanged properly according to the turbo principle we need to perform the EXIT analysis. The analytical derivation of the relationship between the EXIT curves of the component decoders are presented in Sect. 3.

The factor graph of non-systematic ARA decoder with doping is depicted in Fig. 4. The factor graph of the decoder is actually a reverse-version of the encoder's factor graph. Note that, without doping, some of the check nodes has to have degree 1 [22]. Otherwise, the convergence does not start, because there is zero a priori information to be provided for the variable node decoder; this is due to the fact that if one of the check node inputs is zero, the check node output is also zero.

\subsection{Reshaping the Accumulator EXIT Curve}

In order to enhance the convergence, inner systematic doping [19] is used for the outer ACC. To see the difference between inner systematic doping and outer perfect doping reader may check [19]. This mechanism is demonstrated in Fig. 5. The principle is to transmit

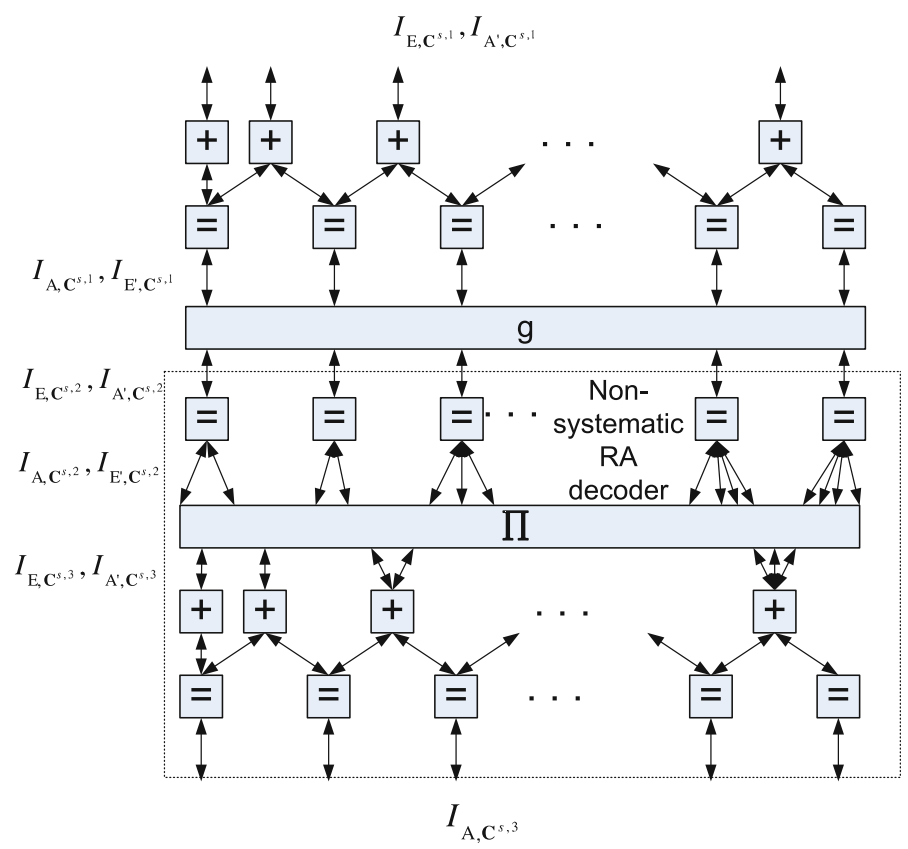

Fig. 4 Factor graph of non-systematic ARA decoder with doping 

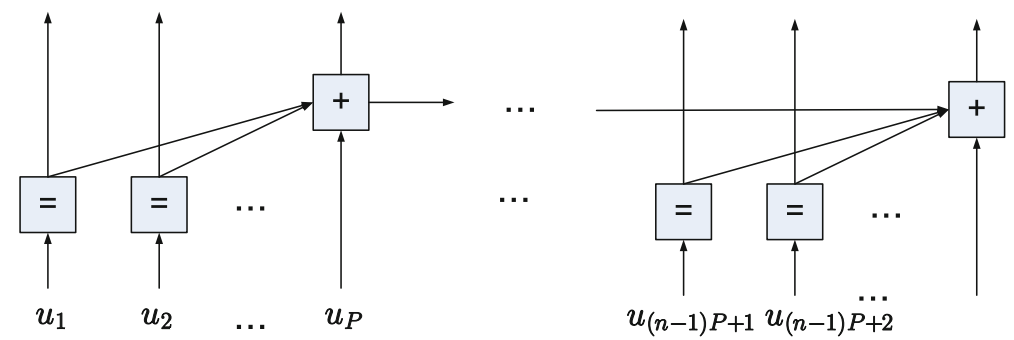

Fig. 5 Factor graph of the doped accumulator

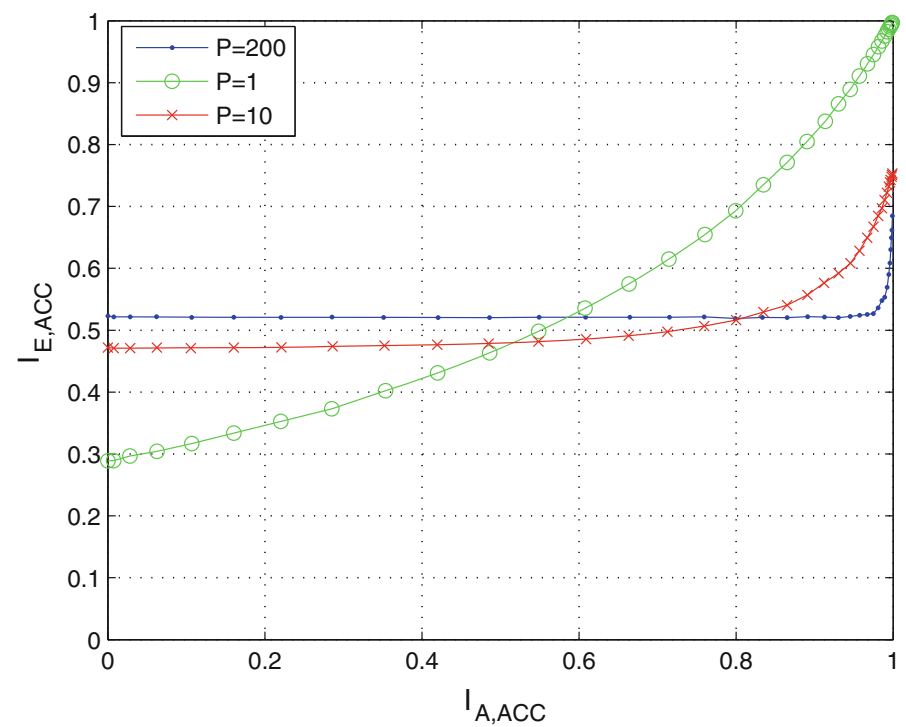

Fig. 6 EXIT chart for accumulator doping with different $P$ values. The ratio between the noise and the energy per generated source bit $E_{s o} / N_{0}=0.5 \mathrm{~dB}$, and the code rate of the RA code is $R_{\mathrm{c}}^{\mathrm{RA}}=0.5$

ACC-coded bits in part, but in the other part directly from the check node output without ACC. With the technique described above, the shape of the EXIT function can be flexibly controlled by choosing the $P$ value, for which the length $L$ sequence is divided to $n$ blocks, so that $n P=L$. Only every $P$ th bit is taken from the accumulator output and the others are taken without accumulation. The effect of this method in terms of the shape of the EXIT curve is presented in Fig. 6. It can be seen that when $P$ increases, MI increases on the left most part of the EXIT curve. Note that because the code rate does not change, the area property [23] justifies the fact that the right most part of the EXIT curve is lower when $P$ increases.

\section{Extrinsic Information Transfer Characteristic}

In order to perform the EXIT analysis for the system we need to identify the relationship between the extrinsic MIs $I_{E, \mathbf{C}^{1,1}}$ and $I_{E, \mathbf{C}^{2,1}}$, which also identifies the relationship between a priori MIs $I_{A^{\prime}, \mathbf{C}^{1,1}}$ and $I_{A^{\prime}, \mathbf{C}^{2,1}}$ due to the symmetry of the system, assumed. Now, we can write the following two EXIT functions: 


$$
\begin{aligned}
& I_{E, \mathbf{C}^{2,1}}^{(l)}=T_{\mathbf{C}^{2,1}}\left(I_{A, \mathbf{C}^{2,1}}^{(l)}, I_{A^{\prime}, \mathbf{C}^{2,1}}^{(l-1)}\right), \\
& I_{A, \mathbf{C}^{2,1}}^{(l)}=g^{-1}\left(I_{E, \mathbf{C}^{2,2}}^{(l)}, I_{A_{\mathbf{D}}^{\prime}}\right)=g^{-1}\left(T_{\mathbf{C}^{2,2}}\left(I_{A^{\prime}, \mathbf{C}^{2,2}}^{(l-1)}, I_{A, \mathbf{C}^{2,2}}^{(l)}\right), I_{A_{\mathbf{D}}^{\prime}}\right),
\end{aligned}
$$

where $l$ is the iteration index, $g$ denotes the EXIT function representing the effect of the use of doping and $I_{A_{\mathbf{D}}}$ indicates the a posteriori MI obtained from the doped bits introduced in Sect. 2.1. Because the MI does not change after interleaving or deinterleaving, following equivalence holds

$$
I_{A, \mathbf{C}^{2,2}}^{(l)}=I_{E, \mathbf{C}^{2,3}}^{(l)}=T_{\mathbf{C}^{2,3}}\left(I_{\left.A, \mathbf{C}^{2,3}, I_{E^{\prime}, \mathbf{C}^{2,2}}^{(l-1)}\right),}\right.
$$

It can be obtained from Fig. 3 that

$$
I_{E^{\prime}, \mathbf{C}^{2,2}}^{(l-1)}=T_{\mathbf{C}^{2,2}}^{\prime}\left(g\left(I_{E^{\prime}, \mathbf{C}^{2,1}}^{(l-1)}, I_{A_{\mathbf{D}}^{\prime}}\right), I_{A, \mathbf{C}^{2,2}}^{(l-2)}\right)
$$

and

$$
I_{E^{\prime}, \mathbf{C}^{2,1}}^{(l-1)}=T_{\mathbf{C}^{2,1}}^{\prime}\left(F_{c}\left(I_{E, \mathbf{C}^{1,1}}^{(l-1)}\right), I_{A, \mathbf{C}^{2,1}}^{(l-2)}\right),
$$

where $F_{c}$ represents the EXIT function corresponding to (5). Substituting (8), (9), (10) and (11) into (7) we know that the extrinsic MI $I_{E, \mathbf{C}^{2,1}}$ depends only on the MI $I_{E, \mathbf{C}^{1,1}}$, channel SNR, bit flipping parameter $p$ and the doping pattern $\mathbf{D}$. This can be expressed as

$$
I_{E, \mathbf{C}^{2,1}}=\tilde{T}_{\mathbf{C}^{2,1}}\left(I_{E, \mathbf{C}^{1,1}}, E_{s o} / N_{0}, p, \mathbf{D}\right),
$$

where $E_{s o} / N_{0}$ denotes the ratio between the energy per generated source bit and the noise power.

The extrinsic MI of VND, $I_{E, \mathbf{C}^{s, 2}}$, is actually a sum of several repetition code outputs. The number of different repetition codes equals the number of different variable node degrees, $D_{\mathrm{v}}$. Therefore, the output of the VND can be calculated as

$$
I_{E, \mathbf{C}^{s, 2}}=\sum_{i=1}^{D_{\mathrm{v}}} a_{\mathrm{v}, i} I_{E, \mathbf{C}_{i}^{s, 2}},
$$

where $I_{E, \mathbf{C}_{i}^{s, 2}}$ denotes the extrinsic information provided by the repetition code $i$. Due to the central limit theorem [24], we can approximate the output LLR distribution as a mixture of $D_{\mathrm{v}}$ Gaussian distributions, where every component has a variance $\tilde{d}_{\mathrm{v}, i} \sigma_{\mathrm{v}}^{2}$, whith $\sigma_{\mathrm{v}}^{2}$ denoting

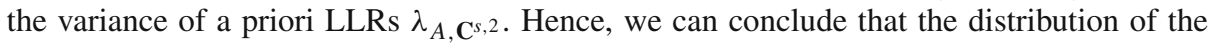
extrinsic LLRs provided by VND can be expressed as

$$
\lambda_{E, \mathbf{C}^{s, 2}} \sim \sum_{i=1}^{D_{\mathrm{v}}} a_{\mathrm{v}, 1} \mathcal{N}\left(\frac{\tilde{d}_{\mathrm{v}, i} \sigma_{\mathrm{v}}^{2}}{2}, \tilde{d}_{\mathrm{v}, i} \sigma_{\mathrm{v}}^{2}\right) .
$$

However, this is true only for the codes which have $R_{\mathrm{C}}^{\mathrm{ARA}} \leq 1$. If $R_{\mathrm{C}}^{\mathrm{ARA}}>1$, the probability distribution of $\lambda_{E, \mathbf{C}^{s, 2}}$ has very high peak at the point where LLR is zero. This happens because as previously mentioned, the check node emits zero if one of the inputs is zero. This breaks the Gaussianity assumption. 


\section{Simulation Results}

In this section, we show the performance of the system for two correlated sources with $p$ as a parameter, which is assumed to be known to the receiver. We will first of all show the EXIT characteristics of the system in high SNR value range. To demonstrate the proper operability of the system as a DJSCC, we then show the performance with low SNR. Simulation parameters are summarized in Table 1. Parameter $R$ denotes the total rate needed to transmit the two sources with arbitrarily low probability of error and $E_{s o}$ is the energy per source bit. $N_{0}$ is zero mean white Gaussian noise with variance $\sigma_{n}^{2}$, which is assumed to be the same in both channels.

\subsection{Correlated Source Compression}

Because the correlation between $\mathbf{U}^{1}=u_{1}^{1}, u_{2}^{1} \ldots$ and $\mathbf{U}^{2}=u_{1}^{2}, u_{2}^{2} \ldots$ is modeled by bit flipping, the SW theorem states that the two sources can be compressed up to the joint entropy $H\left(\mathbf{U}^{1}, \mathbf{U}^{2}\right)=1+H(p)$. In this section, we assume $E_{s o} / N_{0}=10 \mathrm{~dB}$.

The SW sum rate bounds for several $p$ values are shown in Table 2, together with the sum rates required to achieve a point very close to the $(1,1)$ MI point in the EXIT chart by using the proposed method. Performance of turbo code-based technique is also presented for comparison. Only symmetric cases were assumed, i.e., $R_{1}=R_{2}$. The rates were deter-

Table 1 Simulation parameters

\begin{tabular}{|c|c|c|c|c|c|c|c|}
\hline$p$ & $R_{D}$ & $d_{\mathrm{V}}$ & $d_{\mathrm{c}}$ & $a_{\mathrm{c}}$ & $P$ & $L$ & $R$ \\
\hline \multicolumn{8}{|c|}{ (a) Simulation parameters for RA based compression } \\
\hline 0.2 & 0 & 2 & {$\left[\begin{array}{lll}1 & 2 & 3\end{array}\right]$} & {$\left[\begin{array}{llll}0.14 & 0.56 & 0.3\end{array}\right]$} & 1 & 250,000 & 1.85 \\
\hline 0.1 & $9 / 10$ & 2 & {$\left[\begin{array}{ll}1 & 3\end{array}\right]$} & {$\left[\begin{array}{ll}0.1 & 0.9\end{array}\right]$} & 200 & 225,000 & 1.59 \\
\hline 0.05 & $9 / 10$ & 2 & {$\left[\begin{array}{lll}1 & 3 & 4\end{array}\right]$} & {$\left[\begin{array}{lll}0.1 & 0.65 & 0.25\end{array}\right]$} & 0 & 225,000 & 1.46 \\
\hline 0.025 & $9 / 10$ & 2 & {$\left[\begin{array}{lll}1 & 3 & 4\end{array}\right]$} & {$\left[\begin{array}{lll}0.1 & 0.55 & 0.35\end{array}\right]$} & 0 & 225,000 & 1.41 \\
\hline$p$ & $R_{D}$ & $a_{\mathrm{V}}$ & $L$ & $\left(E_{s o} / N_{0}\right)_{\text {prop }}$ & & & \\
\hline
\end{tabular}

(b) Simulation parameters for RA based DJSCC. $R_{\mathrm{C}}=1 / 3$,

$d_{\mathrm{c}}=\left[\begin{array}{lll}1 & 2 & 3\end{array}\right], a_{\mathrm{c}}=\left[\begin{array}{lll}0.2 & 0.2 & 0.6\end{array}\right], d_{\mathrm{v}}=\left[\begin{array}{lll}2 & 4 & 13\end{array}\right]$, and $P=0$ for every $p$

\begin{tabular}{|c|c|c|c|c|}
\hline 0.2 & $9 / 10$ & {$\left[\begin{array}{lll}0.2 & 0.48 & 0.32\end{array}\right]$} & 225,000 & -0.7 \\
\hline 0.1 & $4 / 5$ & {$\left[\begin{array}{lll}0.29 & 0.45 & 0.26\end{array}\right]$} & 200,000 & -1.4 \\
\hline 0.05 & $4 / 5$ & {$\left[\begin{array}{lll}0.29 & 0.45 & 0.26\end{array}\right]$} & 200,000 & -1.9 \\
\hline 0.025 & $4 / 5$ & {$\left[\begin{array}{lll}0.29 & 0.45 & 0.26\end{array}\right]$} & 200,000 & -2.1 \\
\hline 0.01 & $1 / 2$ & {$\left[\begin{array}{lll}0.65 & 0.25 & 0.1\end{array}\right]$} & 125,000 & -2.5 \\
\hline
\end{tabular}

(c) Simulation parameters for RA based DJSCC. $R_{\mathrm{c}}=0.475$, $d_{\mathrm{c}}=\left[\begin{array}{ll}1 & 2\end{array}\right], a_{\mathrm{c}}=\left[\begin{array}{lll}0.2 & 0.2 & 0.6\end{array}\right], d_{\mathrm{V}}=\left[\begin{array}{lll}2 & 4 & 13\end{array}\right]$, and $P=0$ for every $p$

\begin{tabular}{|c|c|c|c|c|}
\hline 0.2 & $9 / 10$ & {$\left[\begin{array}{llll}0.31 & 0.56 & 0.13\end{array}\right]$} & 216,000 & -0.4 \\
\hline 0.1 & $4 / 5$ & {$\left[\begin{array}{llll}0.34 & 0.58 & 0.08\end{array}\right]$} & 192,000 & -1.2 \\
\hline 0.05 & $4 / 5$ & {$\left[\begin{array}{llll}0.34 & 0.58 & 0.08\end{array}\right]$} & 192,000 & -1.8 \\
\hline 0.025 & $4 / 5$ & {$\left[\begin{array}{llll}0.34 & 0.58 & 0.08\end{array}\right]$} & 192,000 & -2.2 \\
\hline 0.01 & $1 / 2$ & {$\left[\begin{array}{llll}0.85 & 0.125 & 0.025\end{array}\right]$} & 120,000 & -2.2 \\
\hline
\end{tabular}


Table 2 The performance of the proposed method with different $p$-values and their corresponding SW limits

\begin{tabular}{lllll}
\hline$p$ & 0.025 & 0.05 & 0.1 & 0.2 \\
$H\left(\mathbf{C}^{1,1}, \mathbf{C}^{2,1}\right)$ & 1.17 & 1.29 & 1.47 & 1.72 \\
$\begin{array}{l}\text { Rate achieved with the } \\
\text { proposed method }\end{array}$ & 1.41 & 1.46 & 1.59 & 1.85 \\
Rate achieved with turbo & 1.31 & 1.435 & 1.63 & 1.89 \\
\hline
\end{tabular}

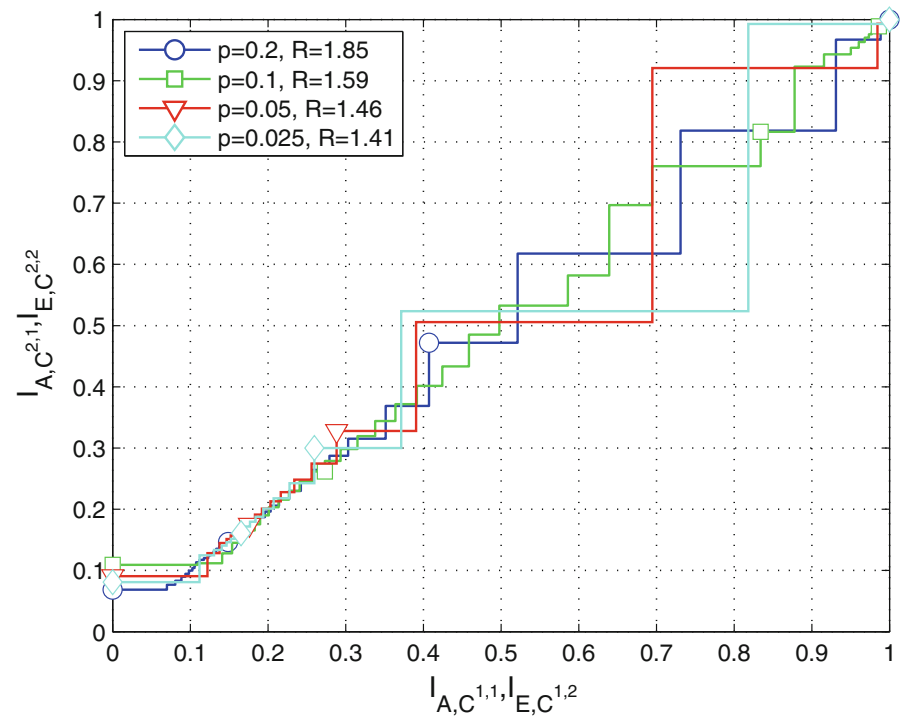

Fig. 7 EXIT trajectories for the proposed compression scheme

mined by adjusting the $R_{D}$ and $d_{\mathrm{c}}$ values. It is found in Table 2 that the proposed system can achieve high compression rate, very close to the SW bound, when the correlation is low. The trajectories shown in Fig. 7 verify that the proposed system achieves a point very close to the $(1,1)$ MI point in the EXIT chart with the parameter values shown in Table 1a.

\subsection{Distributed Joint Source-Channel Coding}

Since we limit our investigations only to 2 -user symmetric systems, we assume that the energy per symbol in both channels is identical, and that $C_{1}=C_{2}=C$, where $C_{i}$ is the capacity of the AWGN channel $i$. In the following, we will use the energy per source bit, $E_{s o}$, which can be related to the energy per information bit, $E_{\mathrm{b}}$, as well as the energy per symbol $E_{\mathrm{s}}$, by [7]

$$
2 E_{s o}=H\left(\mathbf{U}^{1}, \mathbf{U}^{2}\right) E_{\mathrm{b}}=\left(1 / R_{\mathrm{c} 1}+1 / R_{\mathrm{c} 2}\right) E_{\mathrm{s}},
$$

where $R_{\mathrm{c} i}$ is the overall code rate of the encoder for the source $i$, with $i=1,2$. Since we consider the symmetric case only, $R_{\mathrm{c} 1}=R_{\mathrm{c} 2}=R_{\mathrm{c}}$. Hence, with the condition described above,

$$
R<C=\frac{1}{2} \log _{2}(1+S N R),
$$




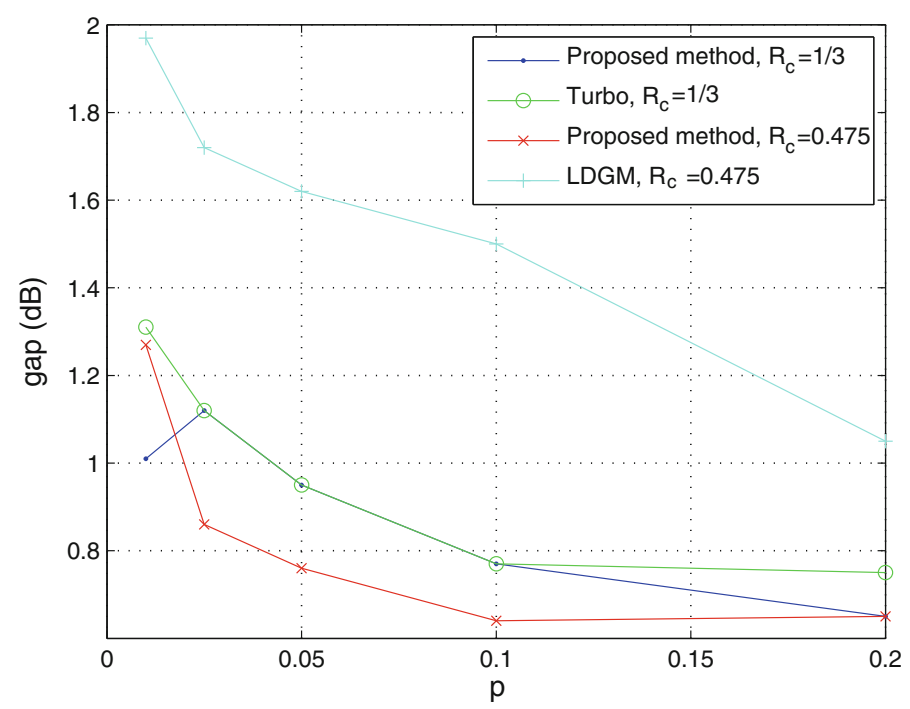

Fig. 8 The gap with respect to the theoretical limit in $\mathrm{dB}$

has to be satisfied. Equation (16) is equivalent to

$$
S N R>2^{2 R}-1,
$$

which leads to the energy per generated source bit to noise power ratio

$$
\begin{aligned}
\frac{E_{\text {So }}}{N_{0}} & >\frac{1}{2 R_{\mathrm{c}}} \frac{E_{\mathrm{s}}}{N_{0}} \\
& =\frac{1}{2 R_{\mathrm{c}}}\left(2^{2 R}-1\right) \\
& =\frac{2^{2 R}-1}{2 R_{\mathrm{c}}} .
\end{aligned}
$$

Furthermore, the overall information rate can be calculated as

$$
R=\frac{H\left(\mathbf{U}^{1}, \mathbf{U}^{2}\right) R_{\mathrm{c}}}{2} \text { information bits per channel use. }
$$

Figure 8 shows the $E_{s o} / N_{0}$ gap in $\mathrm{dB}$ between the theoretical limit and the threshold $E_{s o} / N_{0}$ value at which the proposed method achieves the opening of the convergence tunnel until a point very close to the $(1.0,1.0)$ MI point. Simulations were conducted with various values of $p$ for $R_{\mathrm{c}}=1 / 3$ and $R_{\mathrm{c}}=0.475$. Furthermore, the performances with the symmetric turbo and LDGM based DJSCC are also plotted for comparison. It can be seen that when $R_{\mathrm{c}}=1 / 3, p=0.01$ and $p=0.2$, the threshold $E_{s o} / N_{0}$ is lower with the proposed system than with the turbo DJSCC scheme. An interesting observation is that when $R_{\mathrm{c}}=1 / 3$, we can find a code for $p=0.01$ which achieves the threshold $E_{s o} / N_{0}$ closer to its theoretical limit than the code designed for $p=0.025$. This is because we change $R_{D}$ case by case. Furthermore, when $R_{\mathrm{c}}=0.475$ the proposed scheme can achieve the threshold $E_{s o} / N_{0}$ value much closer to the theoretical limit than LDGM based code proposed in [8] for every value of $p$ considered. Figures 9 and 10 presents the EXIT curves with different $p$ values. We can see that the matching between the trajectories and EXIT curves is almost perfect, even 


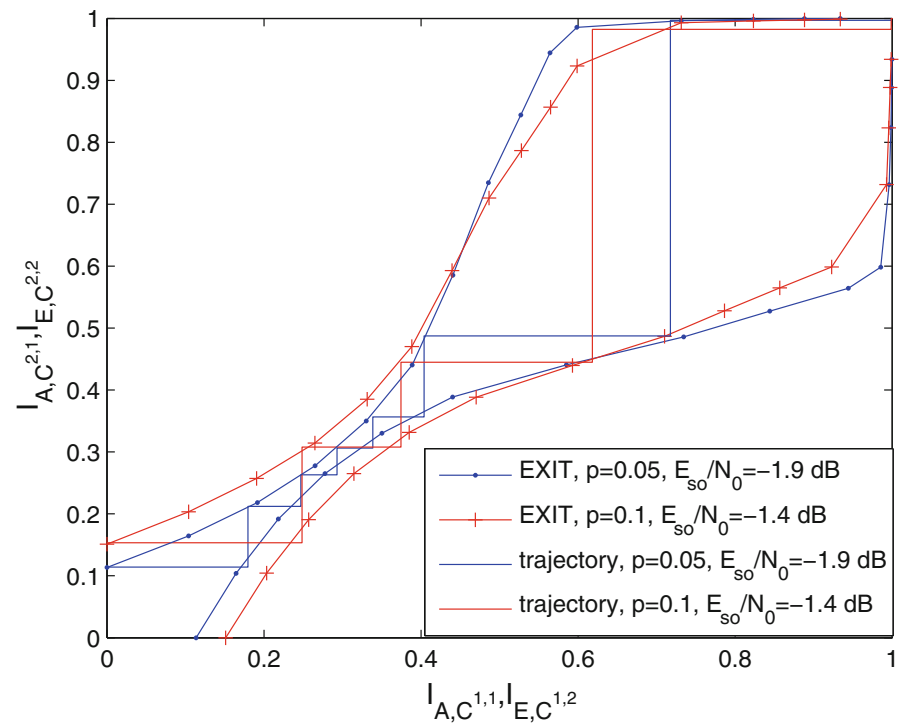

Fig. 9 EXIT chart for proposed DJSCC scheme with $R_{\mathrm{c}}=1 / 3$. Solid line without a marker is the trajectory and the lines with markers are the corresponding EXIT curves

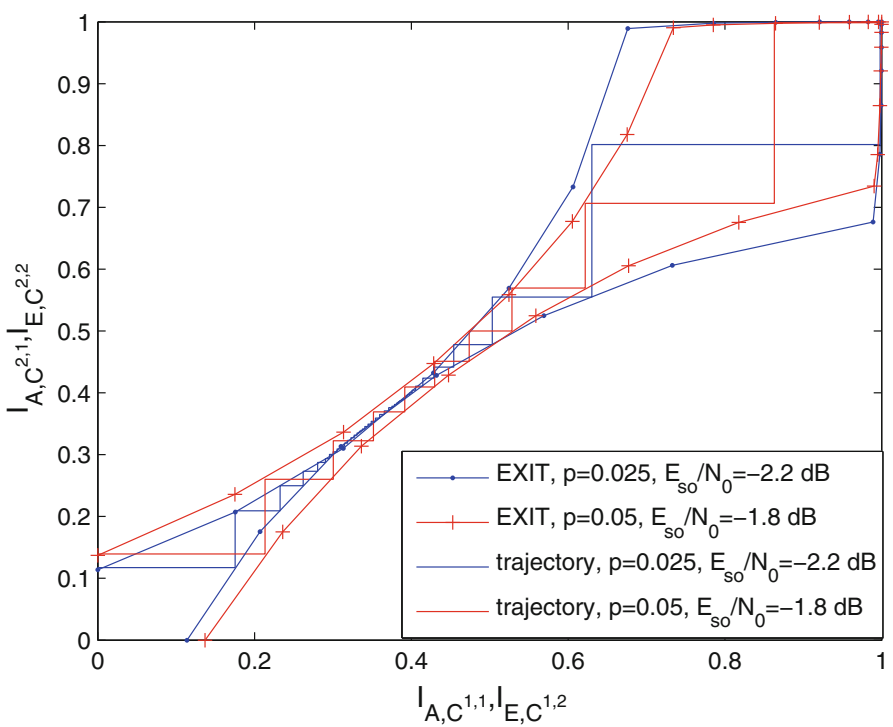

Fig. 10 EXIT chart for proposed DJSCC scheme with $R_{\mathrm{C}}=0.475$. Solid line without a marker is the trajectory and the lines with markers are the corresponding EXIT curves

though slight discrepancies are still observed. This is because the EXIT curves presented here are actually projected EXIT charts of two-fold serially concatenated system, and hence drawing the EXIT curves requires chain simulations for the parts which are concatenated but not mainly focused on [25]. 


\section{Conclusion}

We have proposed a technique for the compression of correlated sources, as an alternative solution to the puncturing based turbo compression techniques. Each source is independently encoded using non-systematic RA based code with the average check node degree larger than the average variable node degree. An advantageous point of the proposed technique over the conventional puncturing-based compression is that no information bits are lost, while they may be lost with the puncturing-based compression, of which category the conventional techniques belong to. The proposed system was also applied to the DJSCC and the performance was compared with turbo and LDGM based DJSCC. It has been observed that the proposed system can achieve better performance in terms of threshold SNR, when the channel code rate is $R_{\mathrm{c}}=1 / 3$ and $R_{\mathrm{c}}=0.475$. This observation should hold also with other values of $R_{\mathrm{c}}$. It should be noted as a concluding statement that extension to the scenarios having more than two sources is left as future study.

Acknowledgments This research was carried out in the framework of the project Distributed Decision Making for Future Wireless Communication Systems (DIDES) which is funded by Finnish Funding Agency for Technology and Innovation (TEKES). This work has been also in part supported by the Japanese government funding program, Grant-in-Aid for Scientific Research (B), No. 23360170. This work has been also supported by Academy of Finland, Riitta ja Jorma J. Takanen Foundation and Finnish Foundation for Technology Promotion.

Open Access This article is distributed under the terms of the Creative Commons Attribution License which permits any use, distribution, and reproduction in any medium, provided the original author(s) and the source are credited.

\section{References}

1. Slepian, D., \& Wolf, J. K. (1973). Noiseless coding of correlated information sources. IEEE Transactions on Information Theory, 19(4), 471-480.

2. Shannon, C. E. (1959). Coding theorems for a discrete source with a fidelity criterion. IRE national convention record (pp. 142-163).

3. Chiang, M., \& Boyd, S. (2004). Geometric programming duals of channel capacity and rate distortion. IEEE Transactions on Information Theory, 50(2), 245-258.

4. Wyner, A. (1974). Recent results in the Shannon theory. IEEE Transactions on Information Theory, 20(1), 2-10.

5. Berrou, C., Glavieux, A., \& Thitimajshima, P. (1993). Near Shannon limit error correcting coding and decoding: Turbo codes. Proceedings of IEEE international conference on communications, Geneva, Switzerland (Vol. 2, pp. 1064-1070).

6. Garcia-Frias, J., \& Zhao, Y. (2001). Compression of correlated binary sources using turbo codes. IEEE Communications Letters, 5(10), 417-419.

7. Garcia-Frias, J., \& Zhao, Y. (2005). Near-Shannon/Slepian-Wolf performance for unknown correlated sources over AWGN channel. IEEE Transactions on Communications, 53(4), 555-559.

8. Garcia-Frias, J., Zhong, W., \& Zhao, Y. (2002). Iterative decoding schemes for source and joint source-channel coding of correlated sources. Proceedings of annual Asilomar conference on signals, systems and computers, Newark, DE, USA (pp. 250-256).

9. Murugan, D. M., Gopala, P. K., \& El Gamal, H. (2004). Correlated sources over wireless channels: Cooperative source-channel coding. IEEE Journal on Selected Areas in Communications, 22(6), 988-998.

10. Gehric, N., \& Dragotti, P. L. (2004). Symmetric and a-symmetric Slepian-Wolf codes with systematic and non-systematic linear codes. IEEE Communications Letters, 9(1), 61-63.

11. Xiong, Z., Liveris, A. D., \& Cheng, S. (2004). Distributed source coding for sensor networks. IEEE Signal Processing Magazine, 21(5), 80-94.

12. Girod, B., Aaron, A. M., Rane, S., \& Rebollo-Monedero, D. (2005). Distributed video coding. Proceedings of IEEE (Invited Paper), 93(1), 71-83. 
13. Rup, S., Dash, R., Ray, N. K., \& Majhi, B. (2009). Recent advances in distributed video coding. Proceedings of IEEE international conference on computer science and information theory, Beijing, China (pp. 130-135).

14. Shamir, G. I., \& Xie, K. (2009). Universal source controlled channel decoding with nonsystematic quick-look-in turbo codes. IEEE Transactions on Communictions, 57(4), 960-971.

15. Divsalar, D., Jin, H., \& McEliece, R. J. (1998). Coding theorems for 'turbo-like' codes. Proceedings of 36th Allerton conference on communication, control and computing, Allerton, IL, USA (pp. 201-210).

16. Gallager, R. (1963). Low-density parity-check codes. Cambridge, MA: MIT Press.

17. Jin, H., \& McEliece, R. J. (1999). RA codes achieve AWGN channel capacity. Applied algebra, algebraic algorithms and error-correcting codes, Pasadena, CA, USA. January 1, 1999 (Vol. 1719/1999, p. 729).

18. ten Brink, S. (1999). Convergence behavior of iterative decoding. IEE Electronics Letters, 35(10), 806-808.

19. ten Brink, S. (2001). Code doping for triggering iterative decoding convergence. Proceedings of IEEE international symposium on information theory, Washington, D.C., USA.

20. ten Brink, S., \& Kramer, G. (2003). Design of repeat-accumulate codes for iterative detection and decoding. IEEE Transactions on Signal Processing, 51(11), 2764-2772.

21. Abbasfar, A., Divsalar, D., \& Yao, K. (2007). Accumulate-repeat-accumulate codes. IEEE Transactions on Communications, 55(4), 692-702.

22. Yano, T., \& Matsumoto, T. (2009). Arithmetic extended-mapping for BICM-ID with repetition codes. Proceedings of ITG workshop smart antennas, Berlin, Germany (pp. 1-8).

23. Ashikhmin, A., Kramer, G., \& ten Brink, S. (2002). Code rate and the area under extrinsic information transfer curves. Proceedings of IEEE international symposium on information theory, Lausanne, Switzerland (p. 115).

24. Peyton, Z., \& Peebles, J. (2001). Probability, random variables, and random signal principles. 1221 Avenue of the Americas, New York, NY, 10020: Irwin/McGraw-Hill.

25. Brännström, F., Rasmussen, L. K., \& Grant, A. J. (2005). Convergence analysis and optimal scheduling for multiple concatenated codes. IEEE Transactions on Information Theory, 51(9), 3354-3364. Proceedings of 2nd international conference on turbo codes, Brest, France (pp. 1-8).

\section{Author Biographies}

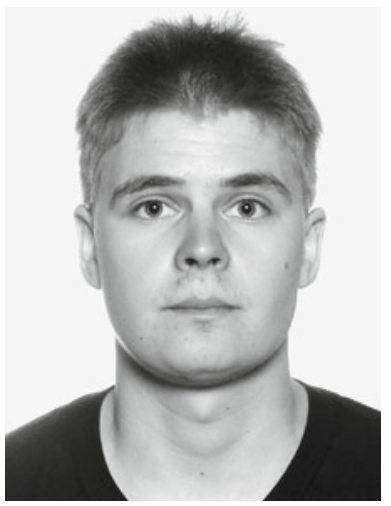

Valtteri Tervo is currently a Ph.D. Student at the Centre for Wireless Communications, University of Oulu, Finland. He is also a doctoral student at the Japan Advanced Institute of Science and Technology (JAIST), Japan. He received his master degree in 2009 in degree program of telecommunications from the University of Oulu. In 2009 he was accepted in the dual degree program between the University of Oulu and JAIST. His research interests are joint source and channel coding, distributed techniques and power allocation based on iterative techniques. 


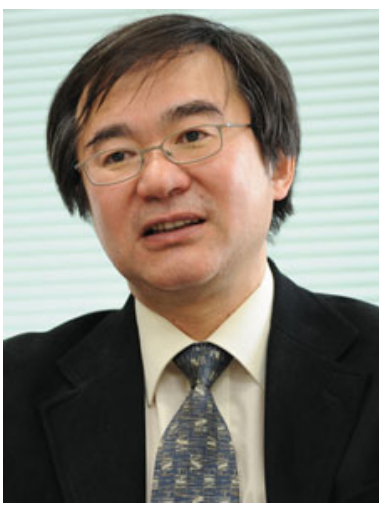

Tad Matsumoto received his B.S., M.S., and Ph.D. degrees from Keio University, Yokohama, Japan, in 1978, 1980, and 1991, respectively, all in electrical engineering. He joined Nippon Telegraph and Telephone Corporation (NTT) in April 1980. Since he engaged in NTT, he was involved in a lot of research and development projects, all for mobile wireless communications systems. In July 1992, he transferred to NTT DoCoMo, where he researched Code-Division Multiple-Access techniques for Mobile Communication Systems. In April 1994, he transferred to NTT America, where he served as a Senior Technical Advisor of a joint project between NTT and NEXTEL Communications.

In March 1996, he returned to NTT DoCoMo, where he served as a Head of the Radio Signal Processing Laboratory. In March 2002, he moved to University of Oulu, Finland, where he served as a Professor at Centre for Wireless Communications. In 2006, he served as a Visiting Professor at Ilmenau University of Technology, Ilmenau, Germany, funded by the German MERCATOR Visiting Professorship Program. In April 2007, he returned to Japan and since then he has been serving as a Professor at Japan Advanced Institute of Science and Technology (JAIST), while also keeping the position at University of Oulu. Prof. Matsumoto has been appointed as a Finland Distinguished Professor for a period from January 2008 thru December 2012, funded by the Finnish National Technology Agency (Tekes) and Finnish Academy, under which he preserves the rights to participate in and apply to European and Finnish national projects. Prof. Matsumoto is a recipient of IEEE VTS Outstanding Service Award (2001), Nokia Foundation Visiting Fellow Scholarship Award (2002), IEEE Japan Council Award for Distinguished Service to the Society (2006), IEEE Vehicular Technology Society James R. Evans Avant Garde Award (2006), and Thuringen State Research Award for Advanced Applied Science (2006), 2007 Best Paper Award of Institute of Electrical, Communication, and Information Engineers (IEICE) of Japan (2008), Telecom System Technology Award by the Telecommunications Advancement Foundation (2009), and IEEE Communication Letters Exemplifying Reviewer Award (2011). He is a Fellow of IEEE. He is serving as an IEEE Vehicular Technology Distinguished Lecturer since July 2011.

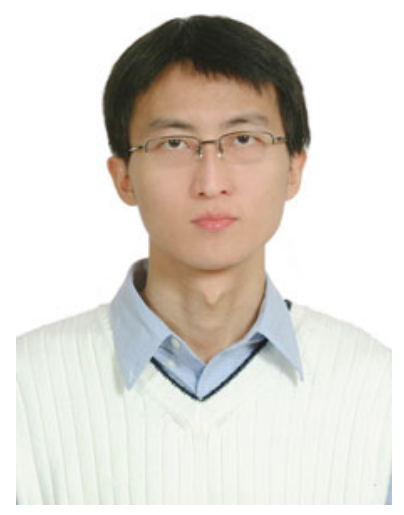

Pen-Shun Lu received the B.S. degree from National Sun Yat-sen University, Taiwan in 2003, and M.S. degree in wireless telecommunication from University of Southampton, UK in 2007. Currently he is a research scientist and perusing his Ph.D. degree in Centre for Wireless Communications (CWC), University of Oulu, Finland. His research interests are in the areas of communications theory, information theory, code theory, and radio communication systems. 